\title{
Mathematical Modeling of Heat Flux Distribution in Raw Cotton Stored in Bunt
}

\author{
Karimov Abdusamat Ismonovich, Ismanov Muhammadziyo \\ Namangan Institute of Engineering and Technology, Namangan, Uzbekistan \\ Email:karimovabdusamat@gmail.com,muhammadziyoismanov39@gmail.com
}

How to cite this paper: Ismonovich, K.A. and Muhammadziyo, I. (2020) Mathematical Modeling of Heat Flux Distribution in Raw Cotton Stored in Bunt. Engineering, 12, 591-599.

https://doi.org/10.4236/eng.2020.128041

Received: July 28, 2020

Accepted: August 17, 2020

Published: August 20, 2020

Copyright (c) 2020 by author(s) and Scientific Research Publishing Inc. This work is licensed under the Creative Commons Attribution International License (CC BY 4.0).

http://creativecommons.org/licenses/by/4.0/

(c) (i) Open Access

\begin{abstract}
The scientific article examines the physical and mechanical properties of raw cotton stored in buntings in cotton palaces. Because during the storage of raw cotton in bunts, some of its properties deteriorate, some improvements. Therefore, the mathematical modeling of storage conditions of raw cotton in bunts and the physical and mechanical conditions that occur in it is of great importance. In the developed mathematical model, the main factor influencing the physical and mechanical properties of raw cotton is the change in temperature. Due to the temperature, kinetic and biological processes accumulated in the raw cotton in Bunt, it can spread over a large surface, first in a small-local state, over time with a nonlinear law. As a result, small changes in temperature lead to a qualitative change in physical properties. In determining the law of temperature distribution in the raw cotton in Bunt, Laplace's differential equation of heat transfer was used. The differential equation of heat transfer in Laplace's law was replaced by a system of ordinary differential equations by approximation. Conditions are solved in MAPLE-17 program by numerical method. As a result, graphs of temperature changes over time in raw cotton were obtained. In addition, the table shows the changes in density, pressure and mass of cotton, the height of the bun. As the density of the cotton raw material increases from the top layer of the bunt to the bottom layer, an increase in the temperature in it has been observed. This leads to overheating of the bottom layer of cotton and is the main reason for the deterioration of the quality of raw materials.
\end{abstract}

\section{Keywords}

Physical, Mechanical, Properties, The Bunt, Parallelepiped,

The Mathematical Model, Biological System, Heat, Processes, Temperature, Coefficient, Experiment, The Bulk Density, Volumetric Density, Humidity, Figure, Coordinate Axes, The Laplace, Differential Equation, Transfer, Solution, Approximate, MAPLE 


\section{Introduction Putting the Issue}

The physical and mechanical properties of raw cotton stored in buntings in cotton palaces differ sharply from their properties in the field. Including:

1) high concentration in limited areas;

2) high density of row cotton in bunt;

3) large in size when placed in the bunt;

4) varying in humidity.

Such cases, do not change the physical state of the cotton pieces, but change its interaction with the external environment. It's known that, how the cotton is stored is of great importance in the initial processing of cotton. Because, during the storage of raw cotton in bunts, some properties deteriorate, some improvements.

Therefore, the mathematical modeling of the conditions of storage of raw cotton in bunts and the physical and mechanical conditions that occur in it is of great importance.

The advantage of mathematical modeling is that, with its help it will be possible to observe the technological properties of raw cotton changes during storage.

The following factors will need to be taken into account when creating the model:

- Change in the density of cotton in the bunt;

- Due to changes in temperature, changes in heat transfer properties;

- In terms of bunt height, compressive strength and relative weight of cotton raw material.

The size of the bunts made in cotton storage palaces is usually $22 \times 11 \times 10 \mathrm{~m}$ and is the form of a parallelepiped. Experiments have shown that the moisture content of the raw material is $9 \%-10 \%$, and the level of pollution is around $5 \%$ $\div 5.5 \%$. The density of raw cotton is around $80 \ldots 110 \mathrm{~kg} / \mathrm{m}^{3}$ in the upper layer and $300-350 \mathrm{~kg} / \mathrm{m}^{3}$ in the lower layer.

In the mathematical model, in order for the thermal conductivity of the raw cotton in the bun to be within a certain norm, the law of maintaining the required thermal insulation and air permeability at a certain size will be required. An increase in density in the lower layer of the cotton bunt weakens the strength of the cotton fibers. This situation, in turn, cannot be explained by the deterioration of the mechanical properties of the fibers. This is because even when the fibers have a density of to $3000 \mathrm{~kg} / \mathrm{m}^{3}$, their mechanical properties do not decrease. It is possible to create a system that changes the physical and mechanical properties of raw materials stored in cotton gins by changing their sensitivity to the external environment, through biological, thermal, chemical and other processes. After the cotton is harvested from the fields, the high heat retention-insulation properties of the fibers in it can be explained by changes in the biological activity of the seed, heat dissipation and the state under which it is pressed into the bunt. Therefore, in the initial processing of cotton, the spontaneous heating of the raw cotton in the bunt and its study is important. 


\section{Mathematical Model of the Problem}

The physical aspect of the problem is that the raw cotton in the bunt, as a biological system, is self-heating. This condition is called self-reorganization.

Self-reorganization:

- Sufficient moisture;

- Immature seeds;

- High heat retention of cotton occurs at the expense.

Seeds of raw cotton form an inversion biological system and have the ability to generate heat and moisture. Cotton harvested from the fields is less fully ripe, leading to varying degrees of generation intensity.

In the mathematical modeling of the problem, we take the parameter of heat release in the biological (seed) system as the cause of the process of self-development in the raw cotton.

The study and analysis of the nature of heat dissipation in raw materials is a key issue in solving the technological problems of storage and primary processing of cotton. Because any change in temperature leads to a change in the basic properties of raw cotton.

For example, due to the accumulated temperature, kinetic and biological processes in the bunt, it can first spread in a small-local state and over time over a large surface with a nonlinear law. As a result, small changes in temperature lead to a qualitative change in the physical properties of raw cotton.

The physical processes that take place in the raw cotton of Bunt are not homogeneous and are subject to non-stationary nonlinear laws.

In addition to the above features, some parameters need to be determined experimentally when building such a mathematical model.

One of them is that the coefficient: $\lambda$ of thermal conductivity of raw cotton on the basis of experiments [1] can be expressed by the following empirical formula:

$$
\lambda=\alpha_{1}+\alpha_{2} T+\alpha_{3} \rho+\alpha_{4} w
$$

Here: T: temperature, $\rho$ : volumetric density, $w$ : humidity, $\alpha_{1}, \alpha_{2}, \alpha_{3}, \alpha_{4}$ : coefficients determined by experiment. For raw cotton, $\rho$ : volumetric density is expressed as follows:

$$
\rho=\rho_{0}\left(1+\frac{\rho^{\prime}}{\rho_{0}}\right)
$$

Here: $\rho$ is the bulk density at the point under test. $\rho_{0}$ is the average bulk density at the test point. $\rho$ 'is the deviation from the average bulk density at the point under test.

We model the temperature distribution in Bunt as follows:

1) Let Bunt be in the form of a rectangular parallelepiped with dimensions $a$, $b, n$ and place the $0 x y z$-coordinate axes as shown in Figure 1 .

2) Let us assume that the heat dissipation in the raw cotton is the same on the surface $(y z)$ and only varies in the vertical $0 x$ direction. That is $(y z)$ isothermal surface; 


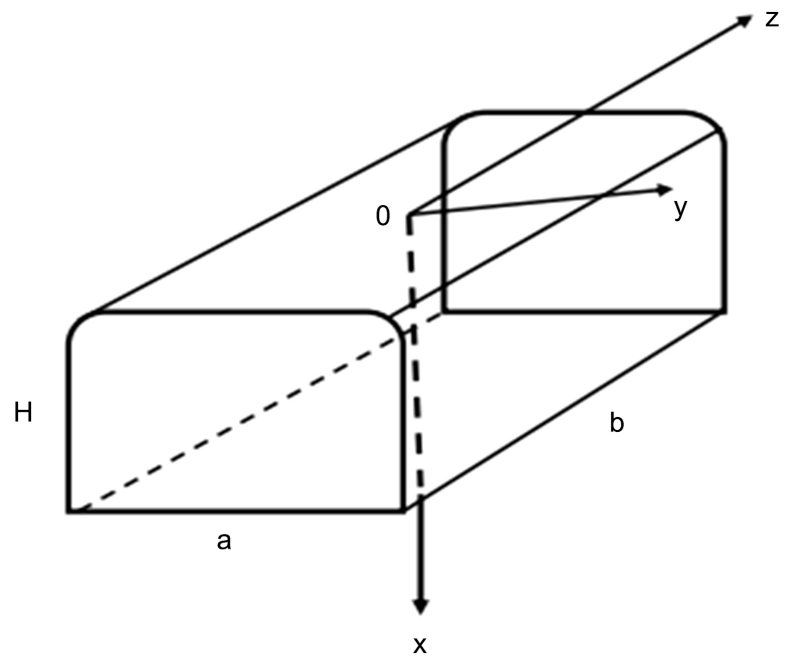

Figure 1. Geometric scheme of the bunt where the raw cotton is stored. a: butting; b: the height of the bunt; $\mathrm{H}$ is the height of the bunt; oxyz: Descartes coordinate system.

3) Let the temperature at any point of the bunt be a function of two variables: $t$. time and $x$. coordinate function, is $T(t, x)$.

Experimental studies [1] [2] have shown that in the hottest part of the bunt, the temperature can rise to $50-80$ degrees, and at points farthest from the center of heating it is around $16^{\circ} \mathrm{C}$ degrees. Humidity is $12 \% \div 14 \%$. Therefore, we can get $T_{\min }=20^{\circ} \mathrm{C}, T_{\max }=50^{\circ} \mathrm{C}$. In this case, if $17^{\circ} \mathrm{C} \leq T \leq 50^{\circ} \mathrm{C}$ changes, we enter the following unmeasured temperature magnitude:

$$
T=T_{0}(1+3 V)
$$

when $T=T_{0}$ to $V=0$; when $T=4 T_{0}$ to $V=1$;

To determine the law of temperature distribution in the raw cotton in Bunt, we use the Laplace differential equation of heat transfer:

$$
\rho c \frac{\partial T}{\partial t}=\Delta(\lambda T)+f(T)-v_{0} \rho c \frac{\partial T}{\partial x}
$$

where $c$ is the heat capacity; $\Delta$ : Laplace operator; $v_{0}$ : rate of diffusion of biological medium activity in self-heating mode; $f(T)$ is a given function; In a special case we get $f(T)=h_{1}\left(T-T_{0}\right), h_{1}$ is the first linear convergence coefficient. We add a nonlinear addition to Equation (4) to keep the energy in equilibrium in the distribution of the temperature of the raw cotton in the bunt without extent $f(T)=-h_{1}\left(T-T_{0}\right)+h_{2}\left(T-T_{0}\right)^{2} ; h_{2}$ is the second linear convergence coefficient. (4) The solution of the differential equation must satisfy the following initial and boundary conditions:

$$
\begin{gathered}
T(0, x)=T(x), \\
T(t, 0)=\varphi_{1}(t), \\
T(t, H)=\varphi_{2}(t)
\end{gathered}
$$

(4): before solving the differential equation, we move on to the following di- 
mensionless quantities: $\bar{x}=\frac{x}{H}, \tau=\frac{t}{t_{0}}, V=V(\tau, \bar{x})$.

Here $H$ is the height of the bunt; $t_{0}$ is the storage time of cotton in the bunt, $V(t, \bar{x})$ is a dimensionless temperature function. We express the relative density of raw cotton in Bunt in linear terms, using the $\bar{x}$ coordinate:

$$
\rho(\bar{x})=\rho_{0}(1+k * \bar{x})
$$

In this case, we write the differential Equation (4), taking into account (1), (3) and (8), in the following dimensionless quantities.

$$
\frac{\mathrm{d} V}{\mathrm{~d} \tau}=f(\bar{x}) \frac{\mathrm{d}^{2} V}{\mathrm{~d} \bar{x}^{2}}-v_{0} \frac{\partial T}{\partial \bar{x}}
$$

Here: $f(\bar{x})=\frac{T_{0}\left(1+4.7 k_{0}+3 k_{0} t_{0}\left(1+k_{0}\right) \bar{x}+1.7 k_{0} x_{0} \bar{x}\right)}{3 t_{0} \rho_{0} c\left(1+k_{1} x_{0} \bar{x}\right)}$

Hence, Equation (9) is the Laplace equation for the temperature distribution over the height of the raw cotton in a cotton ball.

\section{Approximation of the Differential Equation of Heat Transfer}

The problem of heat dissipation in raw cotton is a complex one. It is a boundary problem and it is very difficult to determine its analytical solutions. Therefore, Equation (9) is solved in numerical ways. This method is as follows:

We replace the differential equation with a system of simple differential equations that approximate. To do this, we divide the cotton ball into several layers of the same thickness $(h)$ with horizontal straight lines in height. $x=j^{\star} h$; $j$ : layer number, $h$ : layer width.

In layers formed by the intersection of horizontal lines with the OX-axis (Figure 2), the temperature function depends on a single variable argument $-t$. That is, we define the temperature function in the $j$-layer as follows [2] [3] [4] [5] [6]:

$$
\begin{gathered}
V\left(\tau, x_{j}\right)=V_{j}(\tau) \\
\frac{\partial V\left(\tau, x_{j}\right)}{\partial \tau}=\frac{\mathrm{d} V_{j}(\tau)}{\mathrm{d} \tau} \\
\frac{\mathrm{d}^{2} V\left(\tau, x_{j}\right)}{\mathrm{d} \bar{x}^{2}} \approx \frac{1}{h^{2}}\left[V_{j-1}(\tau)-2 V_{j}(\tau)+V_{j+1}(\tau)\right]
\end{gathered}
$$

Expressions (10)-(12) are approximate calculation formulas. Given (10)-(12), we bring the differential Equation (9) to the following simple first-order system of $n$ differential equations:

$$
\frac{\mathrm{d} V_{j}(\tau)}{\mathrm{d} \tau}=\frac{f_{j}(j h)}{h^{2}}\left[V_{j-1}(\tau)-2 V_{j}(\tau)+V_{j+1}(\tau)\right]-3 T_{0} V_{0} t_{0}\left(V_{j+1}(\tau)-V_{j}(\tau)\right)
$$

Here: $j=1,2,3, \cdots, n ; \quad 0 \leq \tau \leq 1, \quad 0 \leq \bar{x} \leq 1$.

(12): the initial and boundary conditions for the system are written as follows: 


$$
V_{j}(0)=T_{j} ; V_{0}(\tau)=\varphi_{1}(\tau) ; V_{n-1}(\tau)=\varphi_{2}(\tau)
$$

So this is a Cauchy problem. (13): system of first-order differential Equation (14): solved in the MAPLE-17 program under boundary conditions, with graphs of changes in the corresponding temperature, density, compressive strength and cotton mass on the height of the bun, shown in Figure 2, Figure 3, Figure 4. The main quantities used in the calculations in the software program are: $\rho_{0}=80$ $\mathrm{kg} / \mathrm{m}^{3} ; H=10 \mathrm{~m} ; t_{0}=168$ hour; $k=k_{1}=0.25 \mathrm{~m}^{-1} ; c=1.8 \mathrm{Dj} / \mathrm{kg} \cdot{ }^{\circ} \mathrm{C} ; T_{0}=17^{\circ} \mathrm{C}$; $v_{0}=0.0001 \mathrm{~m} / \mathrm{s} ; h_{1}=0.1 \mathrm{Dj} / \mathrm{s} \cdot{ }^{\circ} \mathrm{C} \cdot \mathrm{m}^{3} ; h_{2}=0.2 \mathrm{Dj} / \mathrm{s} \cdot \mathrm{m}^{3} \cdot{ }^{\circ} \mathrm{C}^{2} ; j=1, \cdots, 10 ; \alpha_{1}=0.04 ;$ $\alpha_{2}=0.0004 ; \alpha_{3}=0.00011 ; \alpha_{4}=0.0011$.

\section{Analysis of Results}

As can be seen from the graphs in the figure, the temperature distribution over the height of the bunt varies according to a linear pattern. This change is due to the static law of the initial layers of the bunt, i.e., at $0<x<7 \mathrm{~m}$, and from $x>7$ $\mathrm{m}$ onwards, it grows in the layers close to the bottom of the bunt. The temperature at the top of the bun is the same as the outside temperature $\left(16^{\circ} \mathrm{C}-20^{\circ} \mathrm{C}\right)$, while at the bottom it is around $43^{\circ} \mathrm{C}-48^{\circ} \mathrm{C}$. It is possible to know from the law of change in height. This is because the graphs in Figure 3 show that this coefficient is relatively small at the top and large at the bottom. Figure 4 shows a three-dimensional graph of temperature, bunt height, and time variation. Here, too, the temperature is greater than the bottom layer of the bunt. This leads to spontaneous heating of cotton. In such cases, it is necessary to take appropriate measures to maintain the quality of raw cotton. Table 1 shows the values of the change in density of raw cotton, compressive strength and mass of cotton in terms of bunt height.

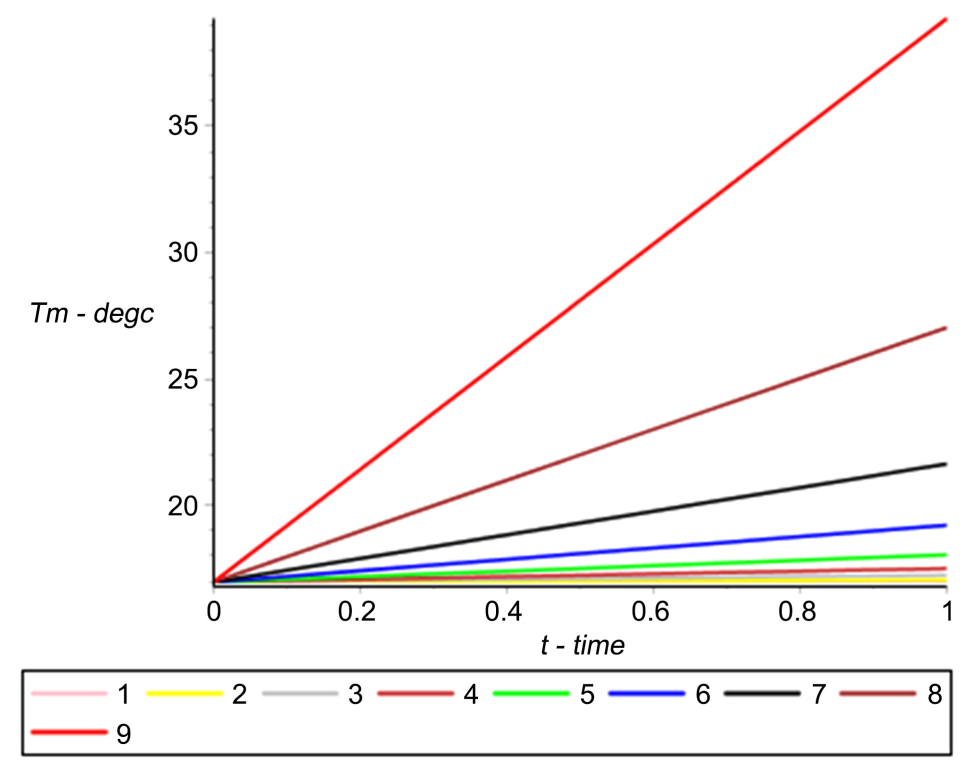

Figure 2. The law of time-arming distribution of temperature $T=T(t, x)$ in the raw cotton in the layers separated by the height of the graphs: $1-x=0.125 ; 2-x=0.250 ; 3-x$ $=0.375 ; 4-x=0.500 ; 5-x=0.625 ; 6-x=0.725 ; 8-x=0.850 ; 9-x=1.0$; e cottonbun. 


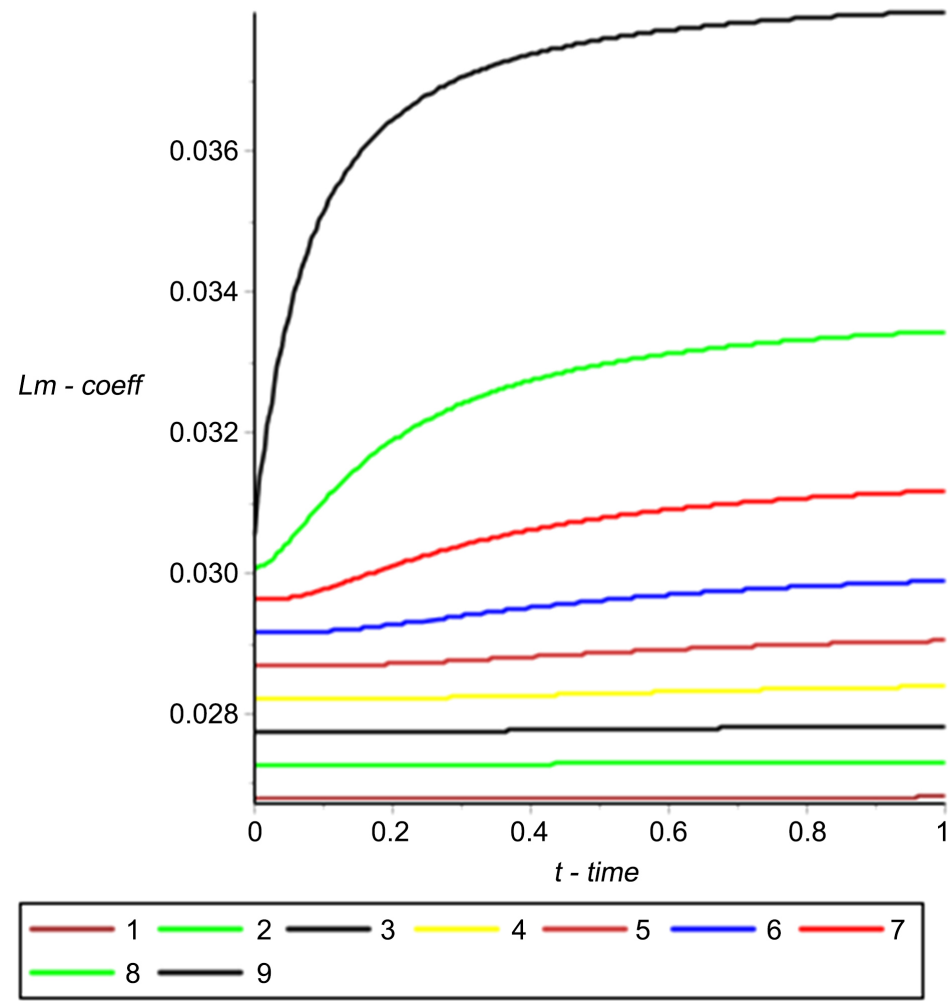

Figure 3. The law of time-varying distribution of the temperature transfer coefficient Lm $=\operatorname{Lm}(t, x)$ in the raw cotton in the layers separated by the height of the cotton bun. In the graphs: $1-x=0.125 ; 2-x=0.250 ; 3-x=0.375 ; 4-x=0.500 ; 5-x=0.625 ; 6-x=$ $0.725 ; 8-x=0.850 ; 9-x=1.0$.

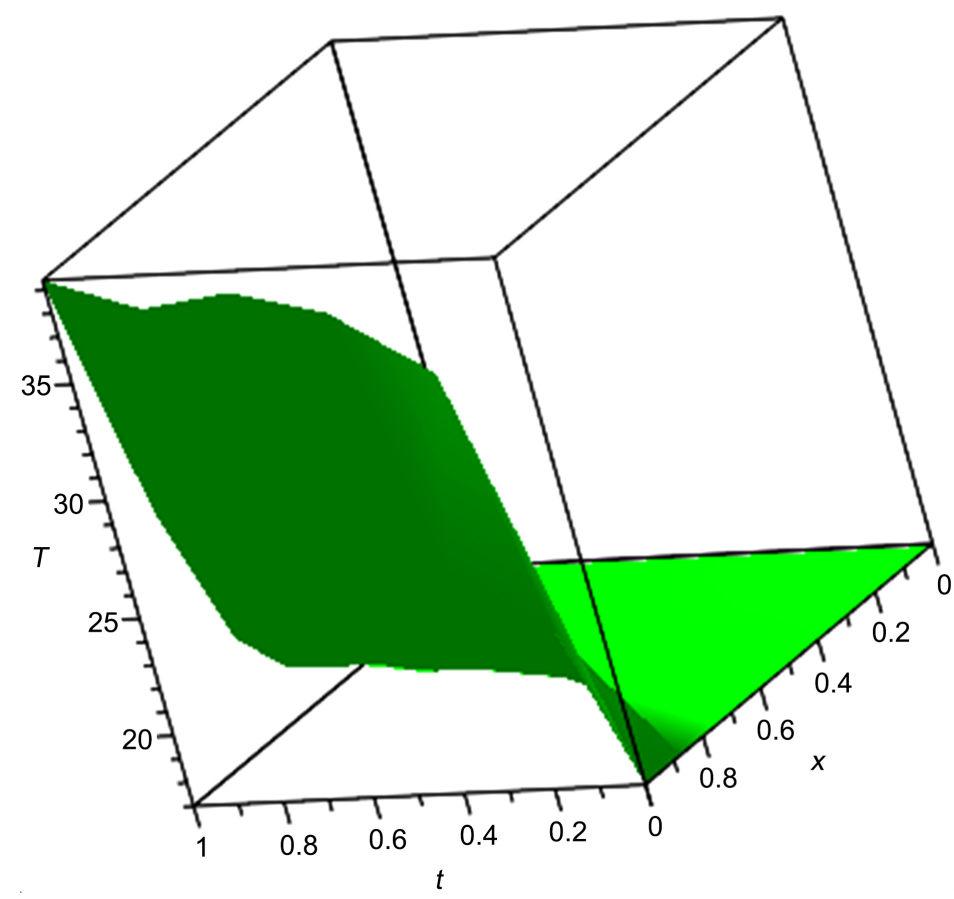

Figure 4. The law of change of temperature $T=T(t, x)$ in the raw material of cotton in the layers separated by the height of the cotton bun depends on the $\mathrm{x}$-coordinate of time and height. 
Table 1. Variation of cotton raw material density, compressive strength and mass according to bunt height. $\mathrm{H}$ is the height of the bunt; $\rho$ : volumetric density; $\mathrm{P}$ : compressive strength; M: mass of cotton.

\begin{tabular}{cccccccccc}
\hline $\mathrm{H}[\mathrm{m}]$ & 0 & 0.125 & 0.250 & 0.375 & 0.500 & 0.625 & 0.750 & 0.875 & 1 \\
$\rho\left[\mathrm{kg} / \mathrm{m}^{3}\right]$ & 100 & 140 & 160 & 180 & 200 & 220 & 240 & 260 & 280 \\
$\mathrm{P}[\mathrm{KPa}]$ & 1 & 4.2 & 6.4 & 9 & 12 & 15.4 & 19.2 & 23.4 & 28 \\
$\mathrm{M}[\mathrm{t}]$ & 24.2 & 101.64 & 154.8 & 217.8 & 290.4 & 372.68 & 464.64 & 566.28 & 677.60 \\
\hline
\end{tabular}

\section{Conclusions}

1) A mathematical model has been developed to determine the temperature changes in cotton, which have a negative impact on the quality of raw cotton stored in the bunt.

2) Laplace's differential heat transfer equation was used to determine the law of temperature distribution in raw cotton in Bunt.

3) The Laplace differential heat transfer equation is solved numerically by MAPLE-17 under the corresponding initial and boundary conditions.

4) The Laplace differential equation of heat transfer is replaced by a system of simple differential equations by approximation.

5) The temperature distribution of the raw cotton in Bunt, the height is divided into 10 layers, and graphs of time change in each layer are obtained.

6) As the density of raw cotton increases from the top layer of the bun to the bottom layer, an increase in temperature is observed. This leads to overheating of the bottom layer of cotton.

7) The changes in density, pressure and mass over the height of the bunt are given in the form of a table.

8) On the basis of the described mathematical model, it is possible to observe the change in temperature at the remaining points of the bunt by means of the temperature value determined at two points in height.

\section{Conflicts of Interest}

The authors declare no conflicts of interest regarding the publication of this paper.

\section{References}

[1] Valiev, F.A. and Nuriev, M.N. (2016) Heat Spreading in the Mass of Raw Cotton during Self-Heating. Science Magazine, Bulletin of Science and Practice, No. 6, 147-151.

[2] Gulyaev, R.A., Lugachev, A.E. and Mardonov, B.M. (2017) Development of an Optimized Technology for Moisturizing Raw Cotton and Studying its Effect on the Preservation of the Natural and Spinning Properties of Cotton Fiber. Journal of Technology of Light Industry Uzbekistan, 1, 15-19.

[3] Tikhonenko, A.B. (2007) Solution of Partial Differential Equations by the Method of Functional Programming in MAPLE. Electronic Scientific Journal, 46, 502-511. 
[4] Ismanov, M.A. and Djo'rayev, Sh.S. (2020) Measuring the Temperature of a Bundle of Cotton Wool. Decision on the Official Registration of the Computer Program.

[5] Ismanov, M., Mardonov, B. and Tadaeva. Y. (2014) Experimental and Theoretical Studies of Vibrational Motion of Raw Cotton on Inclined Mesh Surface. International Journal of Innovation and Scientific Research, 9, 78-85.

[6] Ismanov, M,. Karimov, A. and Azizov, Sh. (2014) Mathematical Modeling of the Technological Processes Original Processing of Cotton. International Journal of Innovation and Applied Studies, 1, 28-39. 